\title{
Correction to: Recommendations for performance optimizations when using GATK3.8 and GATK4
}

Jacob R. Heldenbrand', Saurabh Baheti ${ }^{2}$, Matthew A. Bockol ${ }^{3}$, Travis M. Drucker ${ }^{3}$, Steven N. Hart', Matthew E. Hudson ${ }^{5,9}$, Ravishankar K. Iyer ${ }^{6}$, Michael T. Kalmbach ${ }^{3}$, Katherine I. Kendig ${ }^{1}$, Eric W. Klee ${ }^{4}$, Nathan R. Mattson ${ }^{3}$, Eric D. Wieben ${ }^{7}$, Mathieu Wiepert ${ }^{3}$, Derek E. Wildman ${ }^{8,9}$ and Liudmila S. Mainzer ${ }^{1, *^{*}}$

\section{Correction to: BMC Bioinformatics (2019) 20: 557 https://doi.org/10.1186/s12859-019-3169-7}

Following publication of the original article [1], the author explained that Table 2 is displayed incorrectly. The correct Table 2 is given below. The original article has been corrected.

\begin{abstract}
Author details
${ }^{1}$ National Center for Supercomputing Applications, University of Illinois at Urbana-Champaign, 1205 W. Clark St., Urbana, IL, USA. ${ }^{2}$ Department of Research Services, Mayo Clinic, 200 1st St. SW, Rochester, MN, USA. ${ }^{3}$ Department of IT Executive Administration, Mayo, Clinic, 200 1st St. SW, Rochester, MN, USA. ${ }^{4}$ Department of Health Sciences Research, Mayo Clinic, 200 1st St. SW, Rochester, MN, USA. ${ }^{5}$ Department of Crop Sciences, University of Illinois at Urbana-Champaign, 1102 S. Goodwin Ave., Urbana, IL, USA. ${ }^{6}$ Department of Electrical and Computer Engineering, University of Illinois at Urbana-Champaign, 306 N. Wright St., Urbana, IL, USA. ${ }^{7}$ Department of Biochemistry and Molecular Biology, Mayo Clinic, 200 1st St. SW, Rochester, MN, USA. ${ }^{8}$ Department of Molecular and Integrative Physiology, University of Illinois at Urbana-Champaign, 407 S. Goodwin Ave., Urbana, IL, USA. ${ }^{9}$ Institute for Genomic Biology, University of Illinois at Urbana-Champaign, 1206 W

Gregory Dr., Urbana, IL, USA.
\end{abstract}

Published online: 17 December 2019

\section{Reference}

1. Heldenbrand $J R$, et al. Recommendations for performance optimizations when using GATK3.8 and GATK4. BMC Bioinformatics. 2019;20:557. https:// doi.org/10.1186/s12859-019-3169-7.

\footnotetext{
The original article can be found online at https://doi.org/10.1186/s12859019-3169-7

* Correspondence: Imainzer@illinois.edu

${ }^{1}$ National Center for Supercomputing Applications, University of Illinois at Urbana-Champaign, 1205 W. Clark St., Urbana, IL, USA

${ }^{9}$ Institute for Genomic Biology, University of Illinois at Urbana-Champaign,

1206 W Gregory Dr., Urbana, IL, USA

Full list of author information is available at the end of the article
}

(c) The Author(s). 2019 Open Access This article is distributed under the terms of the Creative Commons Attribution 4.0 International License (http://creativecommons.org/licenses/by/4.0/), which permits unrestricted use, distribution, and reproduction in any medium, provided you give appropriate credit to the original author(s) and the source, provide a link to the Creative Commons license, and indicate if changes were made. The Creative Commons Public Domain Dedication waiver (http://creativecommons.org/publicdomain/zero/1.0/) applies to the data made available in this article, unless otherwise stated. 
Table 2 Splitting the genome by chromosomes

\begin{tabular}{|c|c|c|c|c|c|c|}
\hline Chr & Length & Split 2 & Split 3 & Split 6 & Split 12 & Split 16 \\
\hline 1 & $248,956,422$ & \multirow{9}{*}{1674883629} & \multirow{5}{*}{1061198324} & \multirow{2}{*}{$491,149,951$} & $248,956,422$ & $248,956,422$ \\
\hline 2 & $242,193,529$ & & & & $242,193,529$ & $242,193,529$ \\
\hline 3 & $198,295,559$ & & & \multirow{3}{*}{$570,048,373$} & $198,295,559$ & $198,295,559$ \\
\hline 4 & $190,214,555$ & & & & $190,214,555$ & $190,214,555$ \\
\hline 5 & $181,538,259$ & & & & \multirow{2}{*}{$352,344,238$} & $181,538,259$ \\
\hline 6 & $170,805,979$ & & \multirow{7}{*}{1015844658} & \multirow{3}{*}{$475,290,588$} & & $170,805,979$ \\
\hline 7 & $159,345,973$ & & & & \multirow{2}{*}{$304,484,609$} & $159,345,973$ \\
\hline 8 & $145,138,636$ & & & & & $145,138,636$ \\
\hline 9 & $138,394,717$ & & & \multirow{4}{*}{$540,554,070$} & \multirow{2}{*}{$272,192,139$} & $138,394,717$ \\
\hline 10 & $133,797,422$ & \multirow{15}{*}{1413386203} & & & & $133,797,422$ \\
\hline 11 & $135,086,622$ & & & & \multirow{2}{*}{$268,361,931$} & $135,086,622$ \\
\hline 12 & $133,275,309$ & & & & & \multirow{2}{*}{$247,639,637$} \\
\hline 13 & $114,364,328$ & & \multirow{12}{*}{1011226850} & \multirow{5}{*}{$496,995,021$} & \multirow{3}{*}{$323,399,235$} & \\
\hline 14 & $107,043,718$ & & & & & 209034907 \\
\hline 15 & $101,991,189$ & & & & & $200,004,501$ \\
\hline 16 & $90,338,345$ & & & & \multirow{3}{*}{$253,969,071$} & \multirow{3}{*}{$253,969,07$} \\
\hline 17 & $83,257,441$ & & & & & \\
\hline 18 & $80,373,285$ & & & \multirow{7}{*}{$514,231,829$} & & \\
\hline 19 & $58,617,616$ & & & & \multirow{4}{*}{$220,590,234$} & \multirow{4}{*}{$220,590,23$} \\
\hline 20 & $64,444,167$ & & & & & \\
\hline 21 & $46,709,983$ & & & & & \\
\hline 22 & $50,818,468$ & & & & & \\
\hline $\mathrm{x}$ & $156,040,895$ & & & & \multirow{2}{*}{$213,268,310$} & \multirow{2}{*}{$213,268,310$} \\
\hline$Y$ & $57,227,415$ & & & & & \\
\hline
\end{tabular}

